Алгебра и анализ

Том 19 (2007), № 4
St. Petersburg Math. J. Vol. 19 (2008), No. 4, Pages 597-601

Article electronically published on May 9, 2008

\title{
PONCELET PROBLEM FOR RATIONAL CONICS
}

\author{
V. A. MALYSHEV
}

Abstract. The Poncelet problem for rational conics may have a solution only for polygons with $3,4,5,6,7,8,9,10,12,14,16,18,20$, or 24 vertices.

\section{INTRODUCTION}

In the Poncelet problem, for two conics $A$ and $B$ it is required to construct a polygon whose vertices lie on $A$ and whose sides (or their extensions) are tangent to $B$.

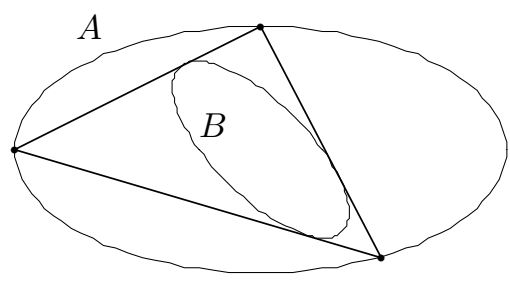

\section{FIGURE 1}

In case the problem is solvable, the Poncelet polygon can start at any point of $A$, and the number of its vertices does not depend on the choice of the initial point [1.

In the present paper we consider conics with rational coefficients. As examples, we mention the conics

$$
\begin{array}{ll}
A: & x^{2}+y^{2}=4, \\
B: & x^{2}+y^{2}=1,
\end{array}
$$

for which it is possible to construct a triangle, the conics

$$
\begin{array}{ll}
A: & x^{2}+y^{2}=4, \\
B: & x^{2}+y^{2}=2,
\end{array}
$$

which admit a quadrangle, and the conics

$$
\begin{aligned}
& A: \quad x^{2}+y^{2}=4, \\
& B: \quad x^{2}+y^{2}=3,
\end{aligned}
$$

which admit a hexagon.

We shall prove that, for conics with rational coefficients, the Poncelet polygons may only have $3,4,5,6,7,8,9,10,12,14,16,18,20$, or 24 vertices. We do not dwell on the

2000 Mathematics Subject Classification. Primary 51N20.

Key words and phrases. Conics.

(C)2008 American Mathematical Society 
problem of constructing a Poncelet polygon for all 15 cases, but exemplify its solution by constructing a pentagon.

\section{§1. Cayley Criterion for the solvability of the Poncelet problem}

The conics $A$ and $B$ can be written in projective coordinates:

$$
\begin{aligned}
& A: \sum_{i, j=1}^{3} A_{i j} x_{i} x_{j}=0, \\
& B: \sum_{i, j=1}^{3} B_{i j} x_{i} x_{j}=0 .
\end{aligned}
$$

We introduce the polynomial

$$
\Delta(t)=\operatorname{det}(t A-B)
$$

and use the coefficients of the Taylor series

$$
\sqrt{\Delta(t)}=c_{0}+c_{1} t+c_{2} t^{2}+c_{3} t^{3}+\cdots
$$

to form the Hankel determinant

$$
H(k, n)=\left|\begin{array}{llll}
c_{k} & c_{k+1} & \ldots & c_{k+n-1} \\
c_{k+1} & c_{k+2} & \ldots & c_{k+n} \\
& & \ldots & \\
c_{k+n-1} & c_{k+n} & \ldots & c_{k+2 n-2}
\end{array}\right| .
$$

By the Cayley criterion, the Poncelet problem is solvable in the class of $(2 n+1)$-gons if and only if

$$
H(2, n)=0,
$$

and is solvable in the class of $(2 n+2)$-gons if and only if

$$
H(3, n)=0 .
$$

For the conics

$$
\begin{array}{ll}
A: & x^{2}+y^{2}=4, \\
B: & x^{2}+y^{2}=1,
\end{array}
$$

we have

$$
\Delta(t)=1-6 t+9 t^{2}-4 t^{3}
$$

and

Therefore,

$$
\sqrt{\Delta(t)}=1-3 t-2 t^{3}-6 t^{4}-\cdots .
$$

$$
H(2,1)=0
$$

and it is possible to construct a triangle.

For the conics

$$
\begin{array}{ll}
A: & x^{2}+y^{2}=2, \\
B: & x^{2}+y^{2}=1,
\end{array}
$$

we have

$$
\Delta(t)=1-4 t+5 t^{2}-2 t^{3}
$$

and

Therefore,

$$
\sqrt{\Delta(t)}=1-2 t-\frac{1}{2} t^{2}-\frac{1}{8} t^{4}-\cdots .
$$

$$
H(3,1)=0
$$


and it is possible to construct a quadrangle.

For the conics

$$
\begin{array}{ll}
A: & x^{2}+y^{2}=4, \\
B: & x^{2}+y^{2}=3,
\end{array}
$$

we have

$$
\Delta(t)=3-10 t+11 t^{2}-4 t^{3}
$$

and

$$
\sqrt{\Delta(t)}=\sqrt{3}\left(1-\frac{5}{3} t+\frac{4}{9} t^{2}+\frac{2}{27} t^{3}+\frac{2}{81} t^{4}+\frac{2}{243} t^{5}-\frac{4}{729} t^{7}-\cdots\right) .
$$

Therefore,

$$
H(3,2)=0
$$

and it is possible to construct a hexagon.

\section{§2. Poncelet problem and Abel equation}

In the paper 2], a relationship was established between the Poncelet problem and the Abel equation. The point is that, for polygons with an even number of vertices, the Cayley solvability criterion for the Poncelet problem is identical to the solvability criterion for the Abel equation in terms of the Hankel determinants 3 .

Suppose $R(x)$ is a polynomial of even degree and without multiple roots. The Abel equation in the ring of polynomials has the form

$$
P^{2}(x)-R(x) Q^{2}(x)=1 .
$$

The trivial solutions $P(x)= \pm 1$ and $Q(x)=0$ are ignored. In the Poncelet problem, the polynomial $R(x)$ will have degree 4 .

With the polynomial

$$
\Delta(t)=a_{0}+a_{1} t+a_{2} t^{3}+a_{3} t^{3}
$$

constructed by the conics $A$ and $B$, we associate the polynomial

$$
R(x)=a_{0} x^{4}+a_{1} x^{3}+a_{2} x^{2}+a_{3} x .
$$

In [3] it was proved that the Abel equation

$$
P^{2}(x)-R(x) Q^{2}(x)=1
$$

has a solution with a polynomial $P(x)$ of degree $n+1$ if and only if

$$
H(3, n)=0 .
$$

Therefore, the Cayley criterion implies that two conics $A$ and $B$ admit a Poncelet $(2 n+2)$-gon if and only if the Abel equation with the polynomial $R(x)$,

$$
P^{2}(x)-R(x) Q^{2}(x)=1,
$$

admits a solution with $P(x)$ of minimal degree $n+1$.

In a similar way, we can prove that the modified Abel equation

$$
P^{2}(x)-R(x) Q^{2}(x)=x
$$

has a solution with $P(x)$ of degree $n+1$ if and only if

$$
H(2, n)=0 \text {. }
$$

In this case, the Abel equation

$$
P^{2}(x)-R(x) Q^{2}(x)=1
$$

has a solution with $P(x)$ of degree $2 n+1$. 
Therefore, the Cayley criterion implies that two conics $A$ and $B$ admit a Poncelet $(2 n+1)$-gon if and only if the Abel equation with the polynomial $R(x)$,

$$
P^{2}(x)-R(x) Q^{2}(x)=1,
$$

admits a solution with $P(x)$ of minimal degree $2 n+1$.

We note that the statement about polygons with an odd number of vertices can be obtained as a consequence of the statement about polygons with an even number of vertices. Indeed, suppose that the conics $A$ and $B$ admit a Poncelet $(2 n+1)$-gon. Then, formally, these conics admit the Poncelet $(4 n+2)$-gon obtained by going around the $(2 n+1)$-gon twice. Therefore, $H(3,2 n)=0$, and the Abel equation with $R(x)$ admits a solution with $P(x)$ of minimal degree $2 n+1$.

\section{§3. Conics with rational coefficients}

Let $A$ and $B$ be conics with rational coefficients. Then the polynomials

$$
\Delta(t)=\operatorname{det}(t A-B)
$$

and

$$
R(x)=x^{4} \Delta\left(x^{-1}\right)
$$

also have rational coefficients.

In [4] it was proved that, for a polynomial $R(x)$ of degree 4 with rational coefficients, the minimal degree of $P(x)$ in the Abel equation

$$
P^{2}(x)-R(x) Q^{2}(x)=1
$$

takes the values

$$
2,3,4,5,6,7,8,9,10,12 .
$$

This allows us to estimate the number of vertices of a Poncelet polygon.

Suppose the Poncelet problem is solvable in the class of $(2 n+2)$-gons. Then

$$
H(3, n)=0 .
$$

Therefore,

$$
n+1=2,3,4,5,6,7,8,9,10,12 .
$$

This means that the corresponding Poncelet polygons may only have

$$
4,6,8,10,12,14,16,18,20,24
$$

vertices. If the Poncelet problem is solvable in the class of $(2 n+1)$-gons, then

$$
H(3,2 n)=0,
$$

whence

$$
2 n+1=3,5,7,9 .
$$

This means that the corresponding Poncelet polygons may have only

$$
3,5,7,9
$$

vertices.

Thus, for conics with rational coefficients, the Poncelet problem can have a solution only for polygons with

$$
3,4,5,6,7,8,9,10,12,14,16,18,20,24
$$

vertices.

Rational conics that admit Poncelet triangles, quadrangles, or hexagons were mentioned in the Introduction. Now we present an example of two rational conics that admit a Poncelet pentagon with rational vertices. 
Example. For the conics

$$
\begin{aligned}
& \text { A : } \quad x^{2}+y^{2}=1 \text {, } \\
& B: 23 x^{2}+18 x-288 y^{2}-576 y=297,
\end{aligned}
$$

the pentagon with the vertices

$$
(1,0),\left(\frac{5}{13},-\frac{12}{13}\right),\left(\frac{3}{5},-\frac{4}{5}\right),(0,-1),\left(-\frac{3}{5},-\frac{4}{5}\right)
$$

is a Poncelet pentagon. Obviously, these points lie on the circle $A$. It is easy to check that the lines going through the corresponding pairs of points are tangent to the hyperbola $B$ at the points

$$
\left(\frac{9}{25},-\frac{24}{25}\right),\left(\frac{27}{59},-\frac{52}{59}\right),\left(1,-\frac{2}{3}\right),\left(1,-\frac{4}{3}\right),\left(-\frac{9}{7},-\frac{8}{7}\right) .
$$

The mutual arrangement of $A$ and $B$ is shown in Figure 2.

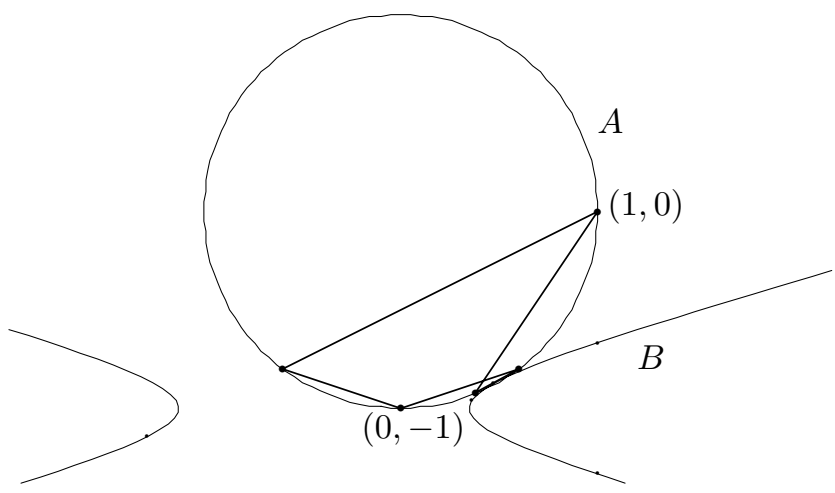

FiguRE 2

The curves $A$ and $B$ intersect at two points, and the pentagon we have constructed has a self-intersection. Only one point of tangency with the hyperbola $B$ lies on a side of the pentagon. The other four tangency points lie on the extensions of sides.

\section{REFERENCES}

[1] P. Griffiths and J. Harris, On Cayley's explicit solution to Poncelet's porism, Enseign. Math. (2) 24 (1978), 31-40. MR0497281 (80g:51017)

[2] V. P. Burskiǐ and A. S. Zhedanov, The Dirichlet problems for the equation of string vibration, the Poncelet problem, the Pell-Abel equation, and some other related problems, Ukrain. Mat. Zh. 58 (2006), no. 4, 435-450; English transl., Ukrainian Math. J. 58 (2006), no. 4, 487-504. MR.2272795 (2008b:35162)

[3] V. A. Malyshev, The Abel equation, Algebra i Analiz 13 (2001), no. 6, 1-55; English transl., St. Petersburg Math. J. 13 (2002), no. 6, 893-938. MR1883839 (2003a:14064)

[4] - Periods of quadratic irrationalities, and torsion of elliptic curves, Algebra i Analiz 15 (2003), no. 4, 177-203; English transl., St. Petersburg Math. J. 15 (2004), no. 4, 587-602. MR2068984 (2005c:11095)

Rybinsk State Aviation Technology Academy, Rybinsk, Russia

E-mail address: wmal@ryb.adm.yar.ru

Received 3/APR/2006

Translated by A. PLOTKIN 